\title{
Urgent and emergent measures in the management of anaphylaxis
}

\begin{abstract}
The word Anaphylaxis is derived from Greek. It is a serious type I hypersensitivity reaction and can be fatal if not recognized and treated with emergency medicines. The lifetime prevalence is $1 \%$ of the population, the most common cause is food related like an egg, peanut, crustaceans, fish, milk tree nuts soya and wheat. It occurs not only by ingestion but also from inhalation and skin contact. It is both Atopic and Non atopic. Diagnosis is only by clinical suspicion and measurement of raised serum Beta Tryptase level can help in supporting the diagnosis.Over the years clinician are experience more cases of anaphylaxis. It is essential that all clinicians should keep adrenaline handy in their medical kits, in clinic, laboratory, every ward, X-ray department and vaccination area. In any doubt of anaphylaxis please inject adrenaline to save a life it won't kill anybody. Most of the developing countries need self-auto injector, Epipen technology at an affordable cost in their countries.
\end{abstract}

Keywords: anaphylaxis, food allergy, adrenaline, epipen, auto injector
Volume I Issue 3 - 2018

\section{Paramesh $\mathrm{H}$}

Department of Pediatric Pulmonologist, Lakeside Center for

Health Promotion, India

Correspondence: Paramesh H, Pediatric Pulmonologist, Environmentalist, Chairman: Lakeside Center for Health Promotion and Lakeside Education Trust, Bangalore, India 560094,Tel 9845022689, Email drhparamesh@gmail.com

Received: February 2, 2018 | Published: May 08, 2018

\section{Introduction}

Allergic diseases are increasing globally. An allergy refers to hypersensitivity due to re-exposure of allergens to sensitized tissues causing the release of inflammatory mediators. Gell and Coombs classified the hypersensitivity disorders of the immune system into four categories like:
i. Type -1 (anaphylaxis),
ii. Type -2 (Antibody cytotoxicity),
iii. Type -3 (Immune complex reaction) and
iv. Type - 4(cellular mediated or delayed hypersensitivity). ${ }^{1}$

Anaphylaxis is a serious typel hypersensitivity reaction and can be fatal if not recognized and treated properly. The word anaphylaxis is derived from Greek Ana=Back; Phylaxis=Immunity. It is first identified by Charles Robert Richer and Paul Portier 116 years ago (1902) who were the French physiologists by experimenting on a dog with the venom of a sea anemone. Anaphylaxis Type 1 reaction has 2 phases, early phase and late phase reactions. Early phase starts in less than $30 \mathrm{~min}$, the late phase can take 2-12 hours. The effector molecule is IgE, the antigens are food, venom, drugs and pollens. The important mediators are histamine, tryptase, leukotriene, prostaglandins, platelet activating factor. The true example of an aphylaxis are anaphylactic reaction, urticaria, allergic rhinitis and allergic asthma. ${ }^{1-3}$ The lifetime prevalence of anaphylaxis from all triggers is $1 \%$ of population and up to $15 \%$ of population are at risk in U.S.A. Food anaphylaxis is the most common cause and $90 \%$ food allergy is from eight items like peanut, egg, crustaceans, milk, fish, tree nuts, soya and wheat. In our country lentils and many legumes contribute for food allergy. ${ }^{3}$

\section{Prevalence of food allergy}

The food allergy is the most common cause of anaphylaxis. The occurence of food allergy in the US and Europe is $2-4 \%$ in adults and 4-8 in children. It is estimated 120,000 emergency room visits less than 200 fatal reactions per year from anaphylaxis. Food allergy anaphylaxis can start from skin contact or by inhalation in allergic people. ${ }^{4-6}$

\section{Mechanism of anaphylaxis'}

1. IgE Mediated: to foods, Insects stings, drugs (NSAID) latex, occupational allergens, aeroallergens, seminal fluid and radio contrast material

2. Non IgE mediated: to radio contrast media, dextrons, biological agents, drugs (NSAIDS)

3. Direct mast cell activation: to physical activity cold, heat sunlight, exercise, drugs (opioids) ethanol

4. Idiopathic: mastocytosis, mast cell activation disorder and some unrecognised allergens.

\section{Factors influencing the anaphylaxis ${ }^{3}$}

a) Age-related factors

i. Infants cannot describe

ii. Mother receiving medicine in labor and delivery to prevent neonatal group B streptococcus infections

iii. In adolescents and young adults who have high risk-taking behaviors

iv. Having concomitant diseases - like asthma, allergic rhinitis, eczema, psychiatry problems (depression)

v. Taking concomitant medicine like alcohol, sedatives, antidepressants, hypnotics, ace inhibitors and beta blockers.

vi. Cofactors which augment the anaphylaxis like acute infections, premenstrual status, exercise and travel which disrupts the routine. 


\section{Diagnosis}

The diagnosis of anaphylaxis is mainly from clinical features as shown in Table 1. One can confirm the diagnosis of anaphylaxis by markedly elevated serum beta-tryptase which is stored in mast cell granules and released explosively in systemic anaphylaxis and the level persists for many hours to few days after fatal anaphylaxis. ${ }^{6,7}$

Table I Any of the three criteria

\begin{tabular}{lll}
\hline Sudden onset of skin and mucus membrane & Sudden respiratory feature & Cardiovascular pressure collapse \\
\hline Urticaria & Cough & Reduced blood pressure Collapse \\
Flushing & Stridor & $30 \%$ reduction of BP Age Specific \\
Itching & Wheeze & $<90 \mathrm{~mm}$ Hg in systolic BP in Adults \\
Swollen lips & Hypoxemia & Others \\
Tongue & Sudden GI symptoms & Incontinence \\
Uvula & Abdominal cramps & \\
& Vomiting & \\
\hline
\end{tabular}

\section{Clinical features of anaphylaxis ${ }^{6}$}

Table 1 Anaphylaxis starts in few minutes to few hours. One has to suspect and treat by clinically the common feature is $90 \%$ starts with skin problem with urticaria, angioedema, itching. 60\% starts with a respiratory problem with stridor or wheeze. $30 \%$ starts with GI symptoms with vomiting, diarrhea. 30\% experience the cardiovascular problems, hypotension with hypoxia. 5.8\% experience nasal symptoms, ocular or palatal pursuits and diaphoresis.

\section{Emergency management of anaphylaxis}

Every hospital should have a written emergency protocol for recognition and treatment of anaphylaxis and practice regularly. Once the patient arrives in the emergency department the physician should assess quickly airway, breathing, circulation, skin, mental status and call for help and inject epinephrine intramuscularly in the midanterolateral aspect of thigh and repeat it in the next $15 \mathrm{~min}$ time and closely follow the patient, most of them respond in 1 or 2 doses. Always place the patient on the back, elevate the legends, and administer humidified oxygen by face mask and establish an intravenous access. If needed cardiopulmonary resuscitation and monitor.

\section{After discharge the following measures to be advised to the patient}

a. Education on how to use epinephrine in an auto-injector, emergency action plan and have a medical identity anaphylaxis alert wrist bend.

b. Confirm the anaphylaxis triggers by specific-skin testing or allergen specific serum IgE level.

c. Avoidance of known triggers, medications and insect stings.

d. Immunotherapy in selected cases by an allergist.

\section{The management guidelines are shown in Table 2}

i. Getting Epipen in India is difficult and very expensive. Hence we made our own arrangement having adrenaline in insulin syringe with needle cover carried inside the small spectacle box like pen as shown in the Figure 1 below and it costs less than one US dollar.

ii. Herewith I am giving you the personal experience of anaphylaxis by the author

iii. I am a known case of allergic rhinitis with occasional seasonal wheeze with exercise induced-bronchospasm mostly post Viral URI since 1975. Not on any regular medicine.

iv. Attended the Rotary district conference dinner in Goa on 29.1.2016, and had rice and Goan fish curry with a single small slice of fish at $10 \mathrm{PM}$.

v. At 2 AM (4 hrs. later) developed intense itching on body and urticaria.
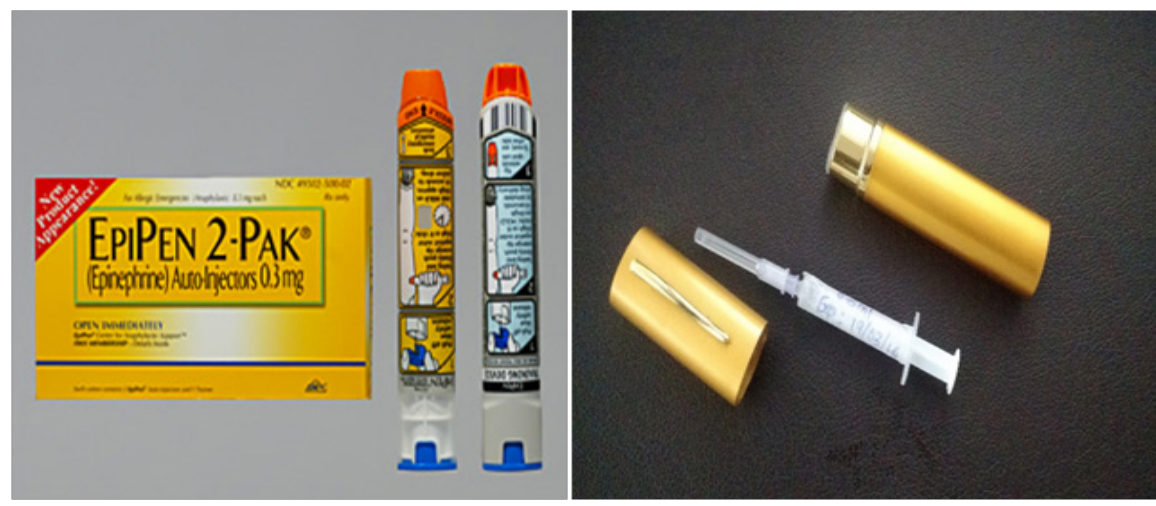

Figure I Adrenaline. 
Table 2 Management of Acute Anaphylaxis ${ }^{8}$

\begin{tabular}{|c|c|c|}
\hline Emergency & Urgency & Be prepared \\
\hline Adrenaline IM $0.01 \mathrm{ml} / \mathrm{kg}$ RPT $-5-15 \mathrm{~min}$ & Humidified $\mathrm{O}_{2}$ & Medical ID Band \\
\hline \multirow[t]{5}{*}{ Adrenaline I-V } & Supine position & Epipen \\
\hline & Elevate legs & Avoid allergen \\
\hline & $\mathrm{B}_{2}$ Agonist inhalation & Immunotherapy \\
\hline & Steroids & \\
\hline & $\mathrm{H}_{2}$ Antihistamines $2^{\text {nd }}$ generation ${ }^{7-9}$ & \\
\hline
\end{tabular}

Self-medicated Allegra 120mg tab (urgent medicine). At 2.15 AM started cough followed by chest tightness very mild wheeze. Took Duolin (Levosabutamol + Ipratropium bromide) 2 puff (urgent medicine). At 2.30 AM, there was swelling of lips. At 2:35 AM developed an urgent need to use toilet to pass stools, (odd time for me). Then I felt dizzy, followed by perspiration on the forehead. At 2.45 AM I woke up my wife and asked her to call hotel reception to take me to take me to the hospital for emergency help. Meantime developed intense chills and cold hands and feet. On the way to hospital developed intense nausea and vomited couple of times and blanked out momentarily and felt going down. Leaned towards the car door making room for lying position and slowly gained conscious and asked people to request the hospital to bring the stretcher then wheelchair. At 3:00 AM people lifted me on a to Stretcher and took me to ER and requested the staff for adrenaline - which was not available (Emergency medicine), they had only first generation antihistamines (Hydroxizine $\mathrm{Hcl}$ ) and steroid (efcorlin) mean while I requested to give $\mathrm{O}_{2}$ and elevate the leg-end of the bed and to start on IV line until doctor arrives. Doctor came in 5 minutes and gave IV Hydroxizine and efcorlin. Later my blood sugar came as $340 \mathrm{mg}$ (Stress related) and Doctor insisted and gave 1 dose of IV insulin and shifted to ICU for observation. In ICU I had sips of water for thirst stayed in ICU for 6 hours. The consultant came at 9AM and after evaluation discharged with oral medicines for allergy + omez for esophagitis and promised to keep the adrenaline all the time in the emergency and the ward. A big thanks to my Wife and the Rotarian friends for their efforts and help. Later it was found out due to fish allergy from elevated serum specific IgE level.

\section{Conclusion}

Anaphylaxis is a serious type I Hypersensitive reaction can be fatal. Food Anaphylaxis is the most common cause and which can come by skin contact or by inhalation of food proteins. $90 \%$ of the time anaphylaxis starts as an acute urticaria, angedema and intense itching. Adrenaline is the drug to be administered and always keep it handy in the clinic and hospitals and adrenaline does not kill anyone in the emergency. Patient should wear medic alert bracelet for identification in an emergency. Always check the content of the food to avoid allergic food. All clinics, hospitals, doctors kits should have adrenaline an emergency medicine. Keep Epipen (Adrenaline) handy. We should influence our stakeholders to bring the technology with cost effectiveness to our needs.

\section{Acknowledgements}

None.

\section{Conflict of interest}

Authors declared that their is no conflict of interest.

\section{References}

1. Asriani M Chiu, Paramesh H. Section Allergy. NELSON essentials of Pediatrics: First South Asian Edition. $1^{\text {st }}$ ed. Asian; 2016:266-283.

2. Cezmi A Akdis. The underlying mechanism in Allergy. Global Atlas of Allergy. EAACI; 2014:39-42.

3. F Estelle $\mathrm{R}$ Simons. Anaphylaxis Global Atlas of Allergy. EAACI;2014:191-196.

4. Susanne Halken, Antonella Muralo. Food Allergy. Global Atlas of Allergy. EAACI; 2014:199-202.

5. Paramesh H. Section 17 Allergy, Partha's 101 clinical pearls in Pediatrics. JAYPEE; 2017:432-452.

6. Mahmoudi, Massoud editors. Challenging cases in allergy and immunology Massoud Mohmoudi. Humana press; 2009.

7. H Paramesh, Nagaraju K, Sukumaran TU, et al Airway diseases education and expertise (ADEX) in Pediatrics. Adaptation for clinical practice in India. Indian Pediatr 2016;53(2):154-158.

8. H Paramesh. Partha's MAPAP. JAYPEE. 2018:180-187.

9. H Paramesh, Nimain C Mohanty, Remesh Kumar, et al. Airway disease education \& expertise (ADEX) next working group recommendations persistent (Chronic) cough in pediatric practice. NIJP. 2017;6(4):230-243. 